\title{
Acknowledgment of Reviewers, 2021
}

The success of Phytobiomes Journal depends on the quality of manuscripts submitted by authors and on the care and competence with which they are reviewed. It is the policy of the Editorial Board to solicit reviews for manuscripts from specialists who are most qualified to review them. In addition to members of the Editorial Board, the individuals listed below provide constructive critical reviews of one or more manuscripts in the past year. Their names are published here in grateful appreciation of their contribution to the journal and to the science of phytobiomes. https://doi.org/10.1094/PBIOMES-6-1-0002

$\begin{array}{lll}\text { Ahmed Abdelfattah } & \text { Holger Heuer } & \text { Ramona Marasco } \\ \text { Erik Alexandersson } & \text { Ann Hirsch } & \text { Mark Mazzola } \\ \text { Artur Alves } & \text { Richard Hogue } & \text { Marco Mechan } \\ \text { Hamed Azarbad } & \text { Mia Howard } & \text { Michael Millican } \\ \text { Matthew Bakker } & \text { Kate Howell } & \text { George Newcombe } \\ \text { R. W. Barreto } & \text { Xiaoping Hu } & \text { Ken Obasa } \\ \text { Justine Beaulieu } & \text { Tzu-Pi Huang } & \text { Paul Ode } \\ \text { Ole Becker } & \text { Carre Isabelle } & \text { Marc Ongena } \\ \text { Terrence Bell } & \text { Ruvishika Jayawardena } & \text { Juan Pablo Frene } \\ \text { Stephane Bellafiore } & \text { Richard Johnson } & \text { Javier Palma-Guererro } \\ \text { Ryan Blaustein } & \text { Lauren Jones } & \text { Tim Paulitz } \\ \text { Tyler Bourret } & \text { Hyun Kim } & \text { Charles Pepe-Ranney } \\ \text { Tomislav Cernava } & \text { William King } & \text { Perrine Portier } \\ \text { Amy Charkowski } & \text { Linda Kinkel } & \text { Haifeng Qian } \\ \text { Elizabeth Cieniewicz } & \text { Stephanie Kivlin } & \text { Hannah Rivedal } \\ \text { Elizabeth Deyett } & \text { Paul Koch } & \text { Caroline Roper } \\ \text { Yongping Duan } & \text { Matthew Kolp } & \text { Daniel Schachtman } \\ \text { Christopher M. M. Franco } & \text { Robert Larkin } & \text { Christopher Schadt } \\ \text { Marc Fuchs } & \text { Seon-Woo Lee } & \text { Adam Schikora } \\ \text { Stefan Geisen } & \text { Johan Leveau } & \text { Dan Schlatter } \\ \text { Deirdre Griffin LaHue } & \text { Steven Lindow } & \text { Angela Sessitsch } \\ \text { Larry Halverson } & \text { Antonino Malacrinò } & \text { Shan Shan } \\ \text { Marjo Helander } & \text { Daniel Manter } & \text { Won-Bo Shim } \\ & & \end{array}$

Marie Simonin

Brajesh Singh

Jane Stewart

Matt Templeton

Anna Testen

Patricia Timper

Andrzej Tkacz

Lindsay Triplett

Mathew Vankoughnett

Patricia Vaz

Entao Wang

Ruoyu Wang

Maggie Warner

Feng Wei

Cesar Xavier

Xiufang Xin

Anthony Yannarell

Carolyn Young

Quan Zeng

Sharon Zytynska 